\title{
Materiales compuestos obtenidos a partir de residuos de raquis de palma africana, alcohol polivinílico y almidón modificado de oca
}

\section{Composite materials based on oil palm empty fruit bunch, polyvinyl alcohol and oca modified-starch}

Presentación: 6-7/10/2020

\section{Doctorando:}

\section{Lauro Vladimir Valle Alvarez}

Centro de Investigaciones Aplicadas a Polímeros, Escuela Politécnica Nacional - Ecuador

Centro Experimental de la Vivienda Económica, Consejo Nacional de Investigaciones Científicas y Técnicas - Argentina Centro de Investigación, Desarrollo y Transferencia de Materiales y Calidad, Facultad Regional Córdoba, Universidad Tecnológica Nacional - Argentina

vladimirvalle81@hotmail.com

\section{Director:}

\section{Jerónimo Kreiker}

\author{
Co-director/a:
}

\section{Belén Raggiotti Francisco Cadena}

\begin{abstract}
Resumen
La gestión de los residuos de raquis generados durante el proceso industrial de extracción de aceite de palma constituye un reto que, en Ecuador, aún no ha sido abordado de manera efectiva. Es así que el objetivo del estudio doctoral es el aprovechamiento de estos residuos en el desarrollo de composites con diferentes matrices poliméricas. En el presente trabajo, se empleó la técnica de moldeo por compresión para la elaboración de materiales compuestos con fibra de raquis, alcohol polivinílico y almidón de oca modificado con urea. Se analizó la influencia de tres factores (temperatura de procesamiento, contenido y tamaño de fibra) sobre el módulo de elasticidad. Adicionalmente, se examinó la presencia de grupos funcionales por espectroscopía infrarroja. Los resultados globales evidenciaron la susceptibilidad de las materias primas para formar composites flexibles y macroscópicamente homogéneos. La evaluación infrarroja mostró varios grupos funcionales característicos de los componentes del material y otros particularmente asociados a carbamatos de almidón de oca. Si bien el factor con significancia estadística para el módulo de elasticidad fue el contenido de fibra, no se observaron correlaciones específicas entre los factores estudiados.
\end{abstract}

Palabras clave: almidón de oca modificado, alcohol polivinílico, raquis de palma, composite

\begin{abstract}
Empty fruit bunch waste management, generating at Ecuadorian oil palm industry, has not been effectively tackled. Thus, the aim of the doctoral study is the use of these residues in composites development with different polymeric matrices. In the present work, compression molding was employed to elaborate composite materials based on empty fruit bunch fiber, polyvinyl alcohol and ureamodified-starch. The influence of three factors (processing temperature, fiber content and size) on modulus of elasticity was analyzed. Additionally, the presence of functional groups was examined by infrared spectroscopy. Overall results showed that raw materials were susceptible to be used in the formulation of flexible and macroscopically homogeneous composites. Infrared evaluation showed several functional groups associated not only to the composite components but also to starch carbamates. Although fiber content showed statistical significance over modulus of elasticity, no specific correlations were observed between the studied factors.
\end{abstract}




\section{Introducción}

La extracción de aceite de palma africana constituye una actividad industrial ampliamente desarrollada en el Ecuador. Durante los últimos diez años, la extracción y comercialización de este producto representó cerca del 4,5\% del producto interno bruto agrícola (Holguín, 2018). El crecimiento de este sector ha traído consigo la generación de una elevada cantidad de desechos sólidos; aquellos relacionados con el raquis constituyen una permanente preocupación ambiental, pues su disposición a cielo abierto en las plantaciones conlleva la proliferación de vectores y alteración de características bioquímicas del suelo.

En la actualidad, uno de los principales retos científico-tecnológicos constituye la valorización de los residuos de raquis en el desarrollo de materiales compuestos con potencial uso en diferentes aplicaciones, pero sobre todo con baja huella de carbono y características compostables. En este sentido, se busca reemplazar las matrices poliméricas tradicionales por opciones como el ácido poliláctico, polihidroxialcanoato, polibutileno succinato, polihidroxibutirato, alcohol polivinílico (PVA), entre otros (Yoon et al., 2012) (Chai et al., 2009). Particularmente, el PVA es un material de bajo costo, versátil y cuya naturaleza hidrofílica favorece la compatibilidad con otros materiales biodegradables (Jayasekara et al., 2004; Rescignano et al., 2014).

En el contexto de la valorización de materias primas naturales y renovables, el almidón es un polisacárido empleado en varias aplicaciones industriales. Sin embargo, debido a ciertas limitaciones en cuanto a su comportamiento mecánico es necesario la modificación estructural por medio de procesos físicos, químicos, enzimáticos y genéticos para alcanzar propiedades avanzadas (Neelam et al., 2012). La modificación química es una de las técnicas más empleadas debido a que permite introducir grupos funcionales en su microestructura. Específicamente, la derivatización con urea promueve la obtención de carbamatos de almidón los cuales proporcionan mayor plasticidad y resistencia al agua (Ferdosian et al., 2017; Menzel et al., 2017a).

El almidón y PVA presentan elevada susceptibilidad para formar mezclas termoplásticas homogéneas (Lu et al., 2009, p. 369) para aplicaciones agrícolas, médicas, así como también para empaque de alimentos (Lu, Xiao y Xu, 2009, p. 370). Si bien el uso y modificación de los almidones ha sido ampliamente estudiado en el desarrollo de materiales compuestos, la mayor parte de estos han sido realizados con especies como maíz, yuca, papa, trigo y arroz (Gadhave et al., 2017, p. 20) y de forma escasa con otras variedades no tradicionales. El almidón de oca (Oxalis tuberosa) con 30 \% de amilosa y 70 \% de amilopectina, proviene de una planta cultivada entre los 2800 y los 4000 metros sobre el nivel del mar en los Andes centrales y meridionales, desde aproximadamente ocho grados latitud norte en Venezuela, hasta 23 grados latitud sur en las regiones septentrionales de Chile y Argentina (Espín et al., 2004).

Desde la perspectiva del aprovechamiento de los residuos generados en extracción industrial de aceite de palma, el objetivo del trabajo doctoral es desarrollar materiales compuestos a partir del residuo de raquis y diferentes matrices poliméricas. Sobre la base de la caracterización de las materias primas se procesarán composites, los cuales serán evaluados en sus principales propiedades con miras a ser aplicados en la industria de la construcción. En este sentido, el presente trabajo evalúa la influencia de la temperatura, contenido y tamaño de fibra en la procesabilidad de un material compuesto elaborado con fibra de raquis, PVA y almidón modificado de oca. Adicionalmente, presenta la caracterización por espectrofotometría infrarroja de los materiales obtenidos y una parte del estudio de comportamiento mecánico.

\section{Desarrollo}

En primer lugar, se realizó la derivatización del almidón nativo de oca con urea en medio de ácido fosfórico conforme el método propuesto por Khalil et. al. (Khalil et al., 2002). En una segunda etapa, se preparó una solución de almidón y PVA; para tal efecto se mezcló 34,5 g de agua, $10 \mathrm{~g}$ de glicerol, $5 \mathrm{~g}$ de etanol y 0,5 g de aceite mineral con agitación constante de $200 \mathrm{rpm}$ por tres minutos. Posteriormente, se agregó $5 \mathrm{~g}$ de PVA y se mantuvo por 30 minutos a $85^{\circ} \mathrm{C}$ bajo agitación constante de $75 \mathrm{rpm}$. Posteriormente, se agregó $35 \mathrm{~g}$ de agua, 0,25 g de benzoato de sodio con $5 \mathrm{~g}$ de almidón de oca modificado.

\section{Preparación del material compuesto}

Se mezcló la fibra de raquis proveniente del pedúnculo de espiguillas con la solución de PVA y almidón modificado de oca, posteriormente, se dejó secar el material a temperatura ambiente por 24 horas. A continuación, se realizó la compresión térmica en una prensa hidráulica marca LAB TECH modelo LP-S-50 a 9 bar por 10 minutos. La preparación 
de los materiales compuestos se realizó sobre la base de un diseño factorial $2^{3}$, dentro del cual se consideró al contenido de fibra, tamaño de fibra ( $\mathrm{N}^{\circ}$ tamiz) y temperatura de prensado como factores de estudio. Los parámetros de formulación de cada una de las muestras se presentan en la Tabla 1.

\begin{tabular}{|c|c|c|c|}
\hline Muestra & ${\text { Temperatura }\left({ }^{\circ} \mathbf{C}\right)}$ & $\mathbf{N}^{\circ}$ tamiz & Contenido de Fibra (\%) \\
\hline M1 & 80 & 40 & 10 \\
\hline M2 & 80 & 40 & 20 \\
\hline M3 & 80 & 30 & 10 \\
\hline M4 & 80 & 30 & 20 \\
\hline M5 & 100 & 40 & 10 \\
\hline M6 & 100 & 40 & 20 \\
\hline M7 & 100 & 30 & 10 \\
\hline M8 & 100 & 30 & 20 \\
\hline
\end{tabular}

Tabla 1: Parámetros de elaboración de materiales compuestos

\section{Caracterización}

Los composites obtenidos fueron evaluados por espectroscopía infrarroja con transformada de Fourier (FTIR) en términos de grupos funcionales característicos, por medio de un espectrofotómetro marca Jasco modelo FT/IR-C800 en modo de reflectancia total atenuada (rango de 4000 a $400 \mathrm{~cm}^{-1}, 25$ barridos y $4 \mathrm{~cm}^{-1}$ de resolución). Complementariamente y sobre la base de la norma ASTM D638 se realizó una evaluación mecánica del módulo de elasticidad por medio de una máquina de ensayos universales marca Instron modelo 3365. Los resultados fueron examinados sobre la base de un análisis de varianza de tres factores. El resumen gráfico del desarrollo metodológico empleado se presenta en la Figura 1.
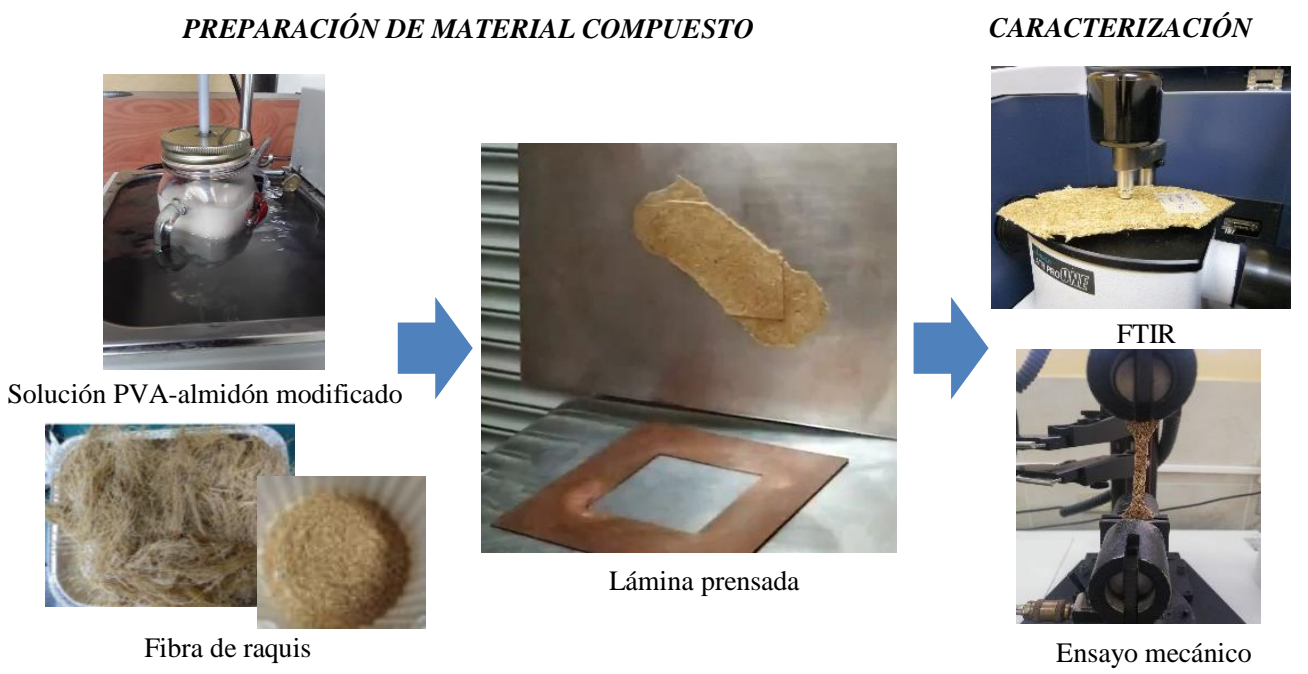

Figura 1: Proceso de obtención y caracterización de materiales compuestos

\section{Resultados}

Los materiales compuestos obtenidos presentaron apariencia relativamente homogénea y flexible, con un espesor de $2,97 \pm 0,26 \mathrm{~mm}$. A nivel macroscópico, las fibras se orientaron totalmente al azar y no se observaron diferencias importantes entre las muestras formuladas. La Figura 2 muestra el registro fotográfico de los materiales obtenidos.

Los resultados del análisis por FTIR, presentados en la Figura 3, evidenciaron varios grupos funcionales importantes en la microestrutura de los materiales compuestos. El primero de ellos, ubicado a $3282 \mathrm{~cm}^{-1}$, se asocia a vibraciones de estiramiento de los grupos hidroxilo $(\mathrm{O}-\mathrm{H})$, los cuales se deben a la contribución del almidón modificado, etanol, glicerol, PVA, así como también de los componentes de la fibra. A $2920 \mathrm{~cm}^{-1}$ se observa una banda atribuida a las vibraciones de tensión del grupo alcano $\mathrm{C}-\mathrm{H}$ (Awada y Daneault, 2015, p.844). La señal a $1727 \mathrm{~cm}^{-1}$ se relaciona con la vibración de tensión del grupo carbonilo $\mathrm{C}=0$ de la hemicelulosa, ceras, PVA y del almidón carbamato, (Nagarkar y Patel, 2019, p.35), 
en tanto que las bandas a $1419 \mathrm{~cm}^{-1}$ y $1368 \mathrm{~cm}^{-1}$ se correlacionan con las vibraciones de flexión y deformación del grupo C-H (Kharazmi et al., 2015, pp.530-531). Adicionalmente, a $1240 \mathrm{~cm}^{-1}$ y $925 \mathrm{~cm}^{-1}$ se presenta el estiramiento de tensión del C-O y la flexión fuera del plano del grupo C-O-H respectivamente (Chen, Li, Zhang y Zhu, 2015, p.4).

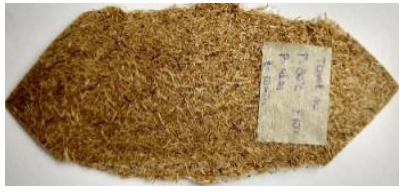

M1

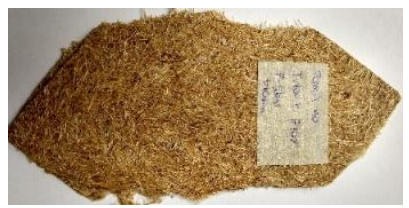

M5

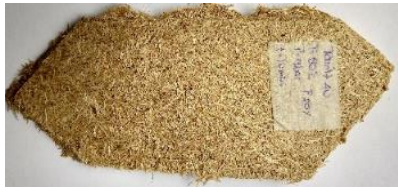

$\mathrm{M} 2$

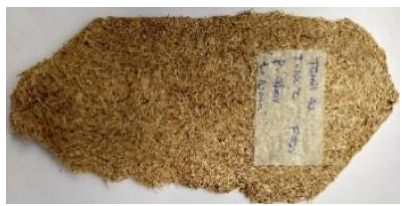

M6

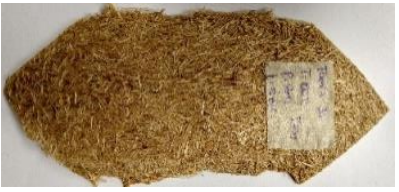

M3

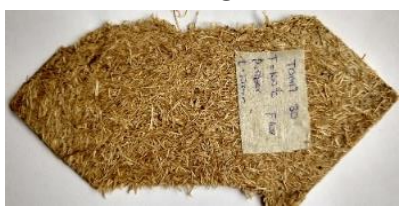

M7

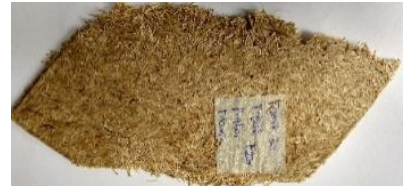

M4

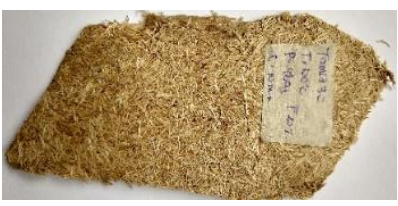

M8

Figura 2: Materiales compuestos elaborados con fibra de raquis, PVA y almidón modificado de oca

Por otra parte, la formación de carbamatos de almidón de oca, producto de la modificación química, se corroboró a través de la presencia del grupo funcional $\mathrm{NH}_{2}$ en dos modos de vibración: flexión de tijera a $1630 \mathrm{~cm}^{-1}$ y aleteo-torsión a $850 \mathrm{~cm}^{-1}$ (Stuart, 2004). De igual forma se evidenciaron varias bandas correspondientes al estiramiento del grupo alifático $\mathrm{C}-\mathrm{N}$ en el rango de $1220 \mathrm{~cm}^{-1}$ a $1020 \mathrm{~cm}^{-1}$ (Siemion et al., 2004).

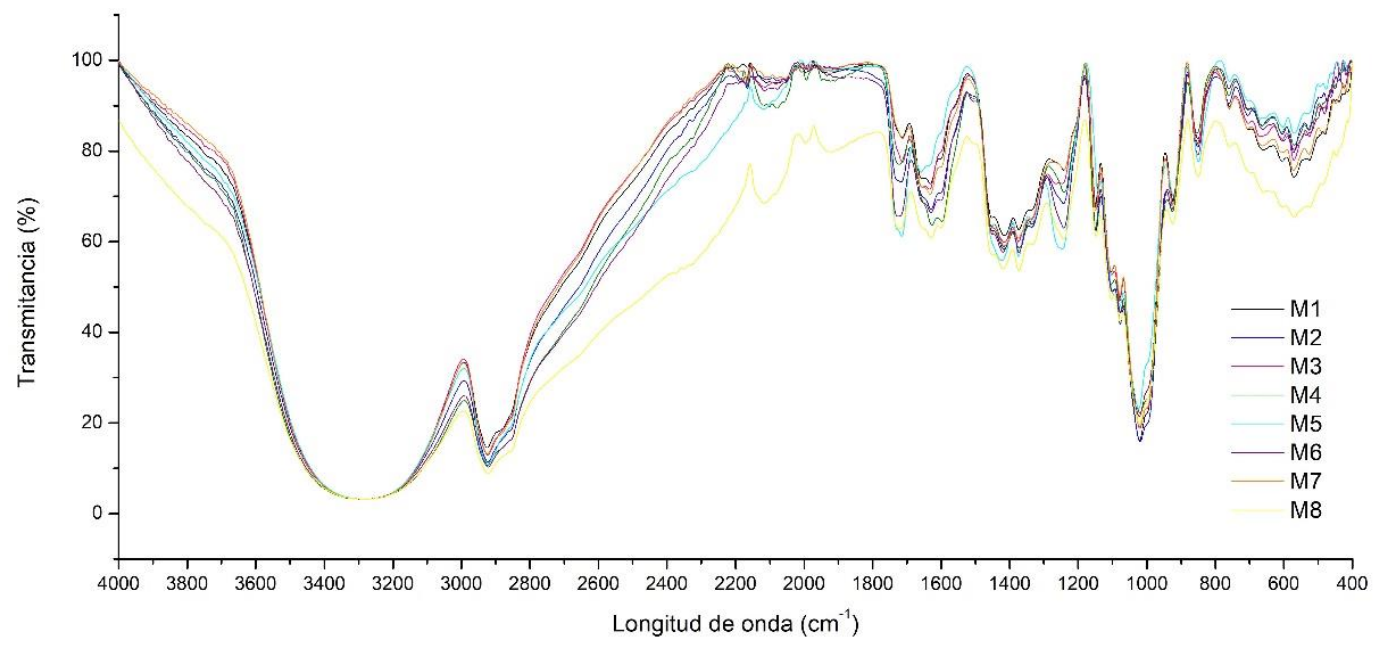

Figura 3: Espectros infrarrojo de materiales compuestos obtenidos

Respecto a la evaluación del módulo elástico, lo resultados de la Figura 4(a) muestran una elevada dispersión de resultados, la cual se debe en primer lugar a la naturaleza heterogénea de las fibras (fases cristalinas y área transversal variable) y en segundo término a la presencia de impurezas y extractivos. Las heterogeneidades presentes en el material compuesto producen concentradores de tensiones los cuales a su vez determinan el comportamiento mecánico de fractura final. Por otra parte, se observa que a mayor cantidad de contenido de fibra aumenta el módulo, lo cual coincide con lo reportado bibliográficamente (Ching et al., 2014). El resumen del análisis de varianza, presentado en la Figura 4(b), evidenció que el factor con significancia estadística es el contenido de fibra. De igual manera, la distribución de los residuos permitió comprobar la suposición de normalidad, es decir que la hipótesis de acercamiento al modelo de Gauss se ha cumplido. Sobre la base de la interacción entre los factores, mostrados en la Figura 4(c) y (d), no se evidenció una correlación específica entre los factores estudiados.

Desde el punto de vista microestructural, la formación de almidón carbamato favorece la capacidad para formar films y el efecto barrera (Menzel et al., 2017a). Dado que el almidón carbamato se obtiene por la reacción del almidón con urea en medio ácido (Khalil, Farag, Aly y Hebeish, 2002, p.255; Menzel et al., 2017, p.367), el producto obtenido incrementa la capacidad de hinchamiento, lo que conduce a una rápida gelatinización (Menzel et al., 2017, p.367; Sun, Yang, Li y Li, 2019, p.460). Sin embargo, un aspecto que no puede ser descartado en esta etapa de la investigación es la posible formación de 
estructuras tridimensionales reticuladas del almidón carbamato, debido a la reacción química de las aminas del grupo carbamato con otras aminas adyacentes y/o a la reacción de las aminas con los grupos $\mathrm{OH}$ de las moléculas de almidón (Khalil et al., 2002, pp.257-260). Este comportamiento puede producirse con cantidades elevadas de urea que inducen la despolimerización debido a la hidrólisis que ocasiona el ácido isociánico, el cual es un producto intermedio en la descomposición de la urea (Passauer y Bender, 2017, pp.358-359). Las reticulaciones producidas aportan rigidez al composite con el consecuente incremento del módulo elástico.

(a)

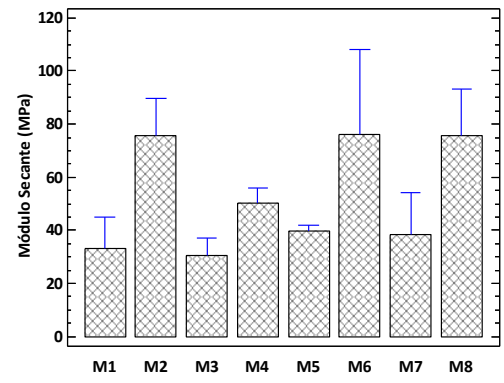

(c)

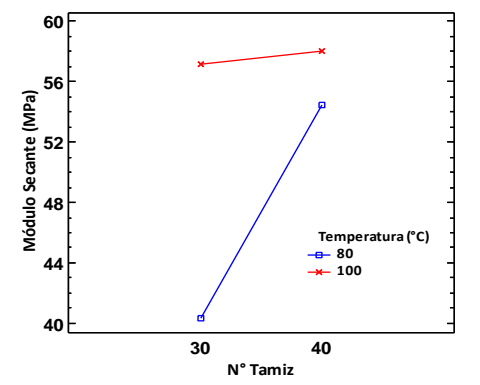

(b)

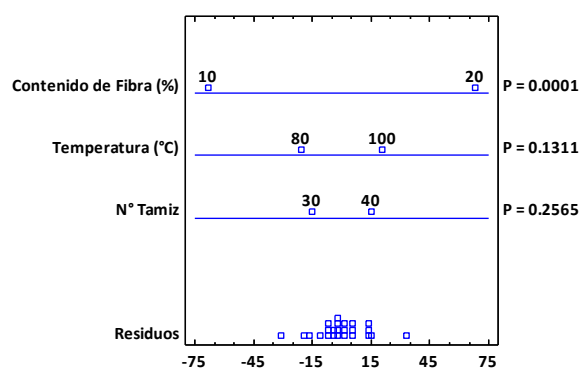

(d)

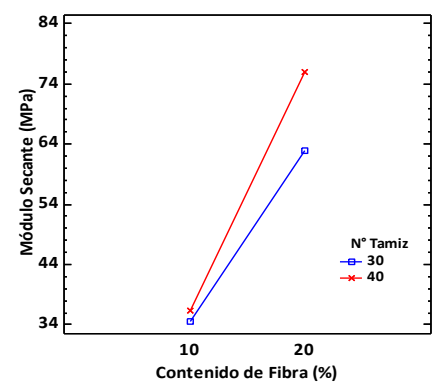

Figura 4: (a) Módulo secante de muestras ensayadas (b) Resumen análisis de varianza (c) Gráfico de interacción temperatura-Nº Tamiz y (d) Gráfico de interacción Nº Tamiz-Contenido de fibra.

\section{Conclusiones}

El almidón modificado de oca, en mezcla con el PVA, es susceptible de ser empleado como materia prima en la elaboración de materiales compuestos con refuerzo de fibras de raquis. Los resultados de la caracterización por FTIR, muestran varios grupos funcionales principalmente relacionados con la presencia de carbamatos de almidón de oca. Por otra parte, el incremento del $10 \%$ de fibra produce un aumento significativo en el módulo elástico. Para el caso de esta propiedad mecánica, el contenido de fibra fue el factor con significancia estadística; sin embargo, el análisis de varianza no refleja correlaciones específicas entre los factores estudiados.

\section{Agradecimientos}

El autor agradece el financiamiento otorgado por la Escuela Politécnica Nacional para ejecución de la investigación, así como también los aportes de Paulina Baquero y Paola Rico en la etapa experimental.

\section{Referencias}

Awada, H., \& Daneault, C. (2015). Chemical modification of poly(vinyl alcohol) in water. Applied Sciences (Switzerland), 5(4), 840-850. https://doi.org/10.3390/app5040840

Chai, W. L., Chow, J. D., Chen, C. C., Chuang, F. S., \& Lu, W. C. (2009). Evaluation of the biodegradability of polyvinyl alcohol/starch blends: A methodological comparison of environmentally friendly materials. Journal of Polymers and the Environment, 17(2), 71-82. https://doi.org/10.1007/s10924-009-0123-1

Chen, J., Li, Y., Zhang, Y., \& Zhu, Y. (2015). Preparation and characterization of graphene oxide reinforced PVA film with boric acid as crosslinker. Journal of Applied Polymer Science, 132(22), 1-8. https://doi.org/10.1002/app.42000 
Ching, K. S., Ealid, M., Ching, Y. C., Haniff, M., Khalid, M., \& Beg, M. T. H. (2014). Preparation and characterisation of polyvinyl alcohol/oil palm empty fruit bunch fibre composite. Materials Research Innovations, 18(sup6), S6-364-S6-367. https://doi.org/10.1109/ICOSP.2014.7015295

Espín, S., Villacrés, E., \& Brito, B. (2004). Caracterización Físico-Química, Nutricional y Funcional de Raíces y Tubérculos Andinos. In V. Barrera, C. Tapia, \& A. Monteros (Eds.), Raíces y Tubérculos Andinos: Alternativas para la conservación y uso sostenible en el Ecuador (Fisrt, p. 30). INIAP/CIP/COSUDE. http://181.112.143.123/bitstream/41000/2827/1/iniapsc322est.pdf

Ferdosian, F., Pan, Z., Gao, G., \& Zhao, B. (2017). Bio-based adhesives and evaluation for wood composites application. Polymers, 9(2). https://doi.org/10.3390/polym9020070

Holguín, A. (2018). Las exportaciones de aceite de palma y su contribución al PIB agrícola de la economía ecuatoriana durante el período 2010-2016. Universidad de Guayaquil.

Jayasekara, R., Harding, I., Bowater, I., Christie, G. B. Y., \& Lonergan, G. T. (2004). Preparation, surface modification and characterisation of solution cast starch PVA blended films. Polymer Testing, 23(1), 17-27. https://doi.org/10.1016/S01429418(03)00049-7

Khalil, M. I., Farag, S., Aly, A. A., \& Hebeish, A. (2002). Some studies on starch-urea-acid reaction mechanism. Carbohydrate Polymers, 48(3), 255-261. https://doi.org/10.1016/S0144-8617(01)00256-9

Kharazmi, A., Faraji, N., Hussin, R., Saion, E., Yunus, W., \& Behzad, K. (2015). Structural, optical, opto-thermal and thermal properties of ZnS-PVA nanofluids synthesized through a radiolytic approach. Beilstein Journal of Nanotechnology, 6(1), 529536. https://doi.org/10.3762/bjnano.6.55

Menzel, C., Seisenbaeva, G., Agback, P., Gällstedt, M., Boldizar, A., \& Koch, K. (2017a). Wheat starch carbamate: Production, molecular characterization, and film forming properties. Carbohydrate Polymers, 172(September), 365-373. https://doi.org/10.1016/j.carbpol.2017.05.053

Menzel, C., Seisenbaeva, G., Agback, P., Gällstedt, M., Boldizar, A., \& Koch, K. (2017b). Wheat starch carbamate: Production, molecular characterization, and film forming properties. Carbohydrate Polymers, 172, 365-373. https://doi.org/10.1016/j.carbpol.2017.05.053

Nagarkar, R., \& Patel, J. (2019). Polyvinyl Alcohol : A Comprehensive Study. Acta Scientific Pharmaceutical Sciences, 3(4), $34-$ 44.

Neelam, K., Vijay, S., \& Lalit, S. (2012). VARIOUS TECHNIQUES FOR THE MODIFICATION OF STARCH AND THE APPLICATIONS OF ITS DERIVATIVES. International Research Journal of Pharmacy, 3(5), 25-31.

Passauer, L., \& Bender, H. (2017). Functional group analysis of starches reacted with urea-phosphoric acid-Correlation of wet chemical measures with FT Raman spectroscopy. Carbohydrate Polymers, 168, 356-364. https://doi.org/10.1016/j.carbpol.2017.03.094

Rescignano, N., Fortunati, E., Montesano, S., Emiliani, C., Kenny, J. M., Martino, S., \& Armentano, I. (2014). PVA bionanocomposites: A new take-off using cellulose nanocrystals and PLGA nanoparticles. Carbohydrate Polymers, 99, 47-58. https://doi.org/10.1016/j.carbpol.2013.08.061

Siemion, P., Jabłonska, J., Kapusniak, J., \& Kozioł, J. (2004). Solid State Reactions of Potato Starch with Urea and Biuret. 12(4).

Stuart, B. H. (2004). INFRARED SPECTROSCOPY: FUNDAMENTALS AND APPLICATIONS (D. J. Ando (ed.)). John Wiley \& Sons Ltd.

Sun, Y., Yang, S., Li, G., \& Li, M. (2019). Preparation of starch phosphate carbamides and its application for improvement of noodle quality. Czech Journal of Food Sciences, 37(6), 456-462. https://doi.org/10.17221/159/2019-CJFS

V. Gadhave, R., Mahanwar, P. A., \& Gadekar, P. T. (2017). Starch-Based Adhesives for Wood/Wood Composite Bonding: Review. Open Journal of Polymer Chemistry, 07(02), 19-32. https://doi.org/10.4236/ojpchem.2017.72002

Yoon, S. Do, Park, M. H., \& Byun, H. S. (2012). Mechanical and water barrier properties of starch/PVA composite films by adding nano-sized poly(methyl methacrylate-co-acrylamide) particles. Carbohydrate Polymers, 87(1), 676-686. https://doi.org/10.1016/j.carbpol.2011.08.046 